\title{
Seroepidemiology of Cytomegalovirus Infection in Pregnant Women in the Central Mexican City of Aguascalientes
}

\author{
Cosme Alvarado-Esquivel ${ }^{\mathrm{a}, \mathrm{d}}$, Maria del Carmen Terrones-Saldivar ${ }^{\mathrm{b}}$, Jesus Hernandez-Tinoco ${ }^{\mathrm{c}}$, \\ Maria Daniela Enriqueta Munoz-Terrones ${ }^{\mathrm{b}}$, Roberto Oswaldo Gallegos-Gonzalez ${ }^{\mathrm{b}}$, \\ Luis Francisco Sanchez-Anguiano ${ }^{\mathrm{c}}$, Martha Elena Reyes-Robles ${ }^{\mathrm{b}}$, \\ Elizabeth Irasema Antuna-Salcidoc
}

\begin{abstract}
Background: Infection with cytomegalovirus (CMV) during pregnancy may lead to congenital disease. Very little is known about the seroepidemiology of CMV infection in pregnant women in Mexico. We sought to determine the seroprevalence and correlates of CMV infection in pregnant women in Aguascalientes City, Mexico.

Methods: Through a cross-sectional study design, 289 pregnant women were examined for anti-CMV IgG and IgM antibodies in Aguascalientes City, Mexico. A standardized questionnaire was used to obtain the socio-demographic, clinical and behavioral characteristics of the pregnant women. The association between CMV infection and the characteristics of the pregnant women was assessed by bivariate and multivariate analyses.
\end{abstract}

Results: Anti-CMV IgG antibodies were detected in 259 (89.6\%) of the 289 pregnant women studied. None of the 289 pregnant women were positive for anti-CMV IgM antibodies. Seroprevalence of CMV infection was significantly lower $(\mathrm{P}=0.03)$ in pregnant women with reflex impairment $(5 / 8: 62.5 \%)$ than in those without this clinical feature (246/272: 90.4\%). Seroprevalence of CMV infection was significantly higher $(\mathrm{P}=0.03)$ in pregnant women with 2 - 9 pregnancies (140/150: $93.3 \%)$ than in those with only one pregnancy $(119 / 139$ : $86.2 \%$ ). Logistic regression analysis of socio-demographic and behavioral variables showed that seropositivity to CMV was associated with contact with children (odds ratio $(\mathrm{OR})=3.56$; $95 \%$ confidence interval (CI): 1.17 - 10.84; $\mathrm{P}=0.02)$, whereas high $(>150 \mathrm{AU} / \mathrm{mL})$ anti-CMV antibody levels were negatively associated with washing

Manuscript submitted January 12, 2018, accepted February 1, 2018

aBiomedical Research Laboratory, Faculty of Medicine and Nutrition, Juarez University of Durango State, Avenida Universidad S/N, 34000 Durango, Mexico

${ }^{b}$ Centro de Ciencias de la Salud, Universidad Autonoma de Aguascalientes, Mexico

'Institute for Scientific Research "Dr. Roberto Rivera-Damm", Juarez University of Durango State, Avenida Universidad S/N, 34000 Durango, Mexico ${ }^{\mathrm{d}}$ Corresponding Author: Cosme Alvarado-Esquivel, Laboratorio de Investigacion Biomedica, Facultad de Medicina y Nutricion, Avenida Universidad S/N, 34000 Durango, Dgo, Mexico. Email: alvaradocosme@yahoo.com

doi: https://doi.org/10.14740/jocmr3358w hands before eating $(\mathrm{OR}=0.17 ; 95 \% \mathrm{CI}: 0.05-0.63 ; \mathrm{P}=0.007)$.

Conclusions: We found a high endemicity of CMV infection in pregnant women in Aguascalientes City, Mexico. Factors associated with CMV infection found in this study may be useful for an optimal planning of preventive measures against CMV exposure in pregnant women.

Keywords: Cross-sectional study; Seroprevalence; Cytomegalovirus; Infection; Pregnant women; Epidemiology; Mexico

\section{Introduction}

Human cytomegalovirus (CMV) is a double-stranded DNA virus, and a member of the Herpesviridae family [1, 2]. Infections with CMV are common worldwide [3]. Primary infection with CMV may lead to lifelong latency in the host with reactivation during periods of relative immunosuppression [1, 2]. Principal sources of CMV infection during pregnancy are young children and intimate contacts [3,4]. Primary infection with CMV occurs in approximately $1-4 \%$ of pregnancies [3]. Congenital transmission of CMV can occur with maternal primary infection, reactivation, or reinfection during pregnancy [5]. Congenital CMV transmission rates are as high as $50 \%$ in women who acquired primary CMV infection during pregnancy, and less than $2 \%$ in women with non-primary infection [3]. Intrauterine CMV transmission rates for primary and non-primary infections are about $30 \%$ and $0.2 \%$, respectively [6]. CMV can also be transmitted from mother-to-child intrapartum or during breastfeeding [3]. Primary CMV infection is mostly asymptomatic in immunocompetent adults; however, infection may manifest as a mild mononucleosis or flu-like syndrome with persistent fever and fatigue [3]. Congenital CMV infection represents a relevant cause of deafness and neurological damage in newborns [6, 7]. In addition, congenital CMV infection may lead to intrauterine growth retardation, microcephalia, petechiae, jaundice, hepatosplenomegaly, and ophthalmological disorders [8]. Infection with CMV can also be transmitted by blood transfusion [9], and organ transplantation [10].

The seroepidemiology of CMV infection in Mexico has 


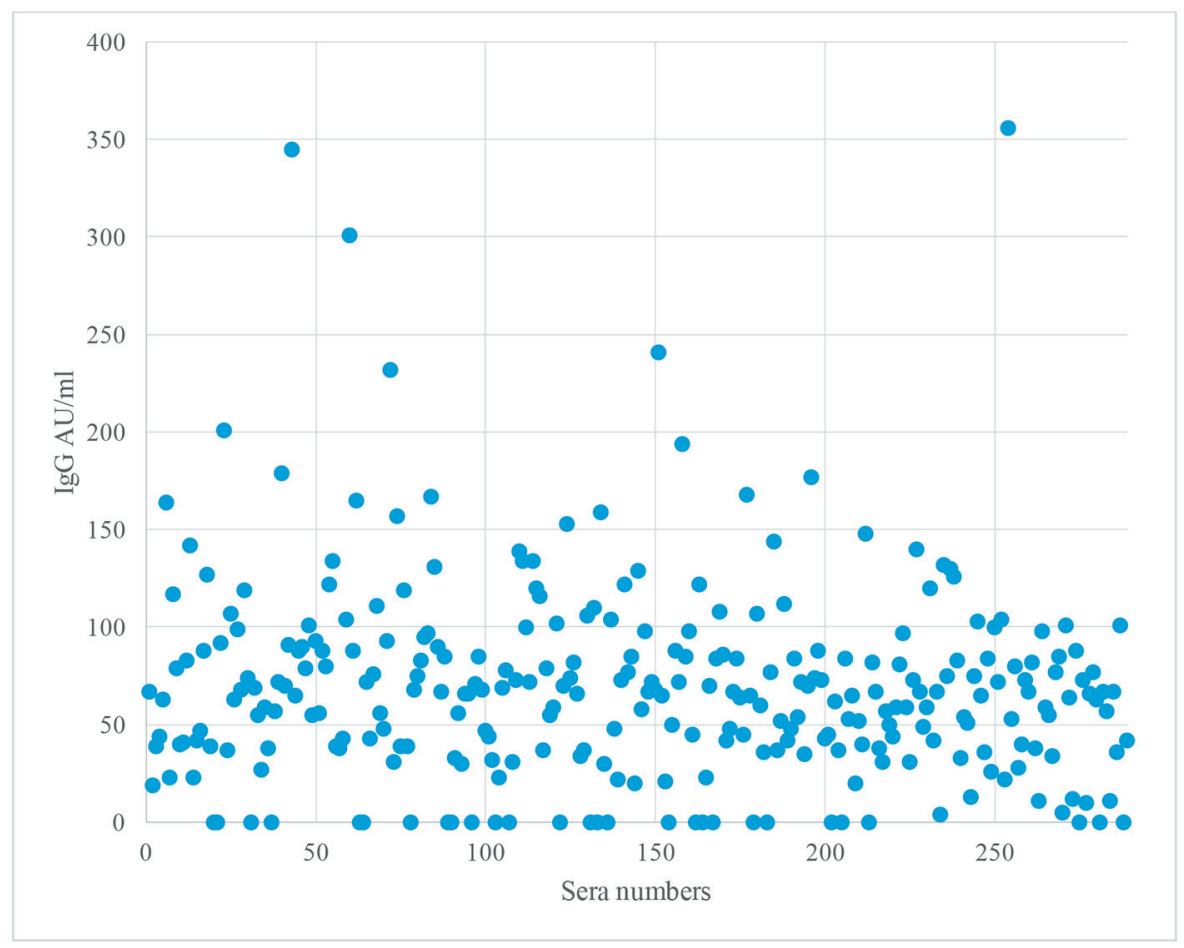

Figure 1. Individual values of anti-CMV IgG antibodies obtained in the 289 pregnant women studied.

been scantily studied. We reported the seroepidemiology of $\mathrm{CMV}$ infection in pregnant women in the northern Mexican state of Durango [11]. In the present study, we sought to determine the seroprevalence and correlates of CMV infection in pregnant women in the central Mexican city of Aguascalientes.

\section{Materials and Methods}

\section{Study design and women studied}

We performed a cross-sectional study of pregnant women in Aguascalientes City, Mexico, from October 2014 to February 2016. Participants were enrolled consecutively in the study when attending their prenatal care consultations in three public health centers (Instituto de Servicios de Salud del Estado de Aguascalientes). Inclusion criteria for enrollment in the study were: 1) pregnant women at their 1 - 9 months of pregnancy; 2) aged 13 - 45 years old; and 3) who voluntarily accepted to participate in the study. Pregnant women were invited to participate in the study regardless of their socio-economic status, occupation, or educational level. In total, 289 pregnant women (mean age: $22.95 \pm 6.29$; range $13-42$ years) were included in the survey.

\section{Socio-demographic, clinical, behavioral and housing char- acteristics of the pregnant women}

We obtained the socio-demographic, clinical, behavioral, and housing characteristics from the pregnant with the aid of a standardized questionnaire in face-to-face interviews. Sociodemographic items included age, ethnic group, birthplace, residence, occupation, educational level, and socio-economic status. Clinical characteristics included obstetric history (month of pregnancy, number of pregnancies, deliveries, cesarean sections and miscarriages), presence of any underlying disease, presence or history of lymphadenopathy, frequent headaches and impairments of memory, reflexes, vision and hearing, history of blood transfusions, hepatitis, surgery or transplants. Behavioral items included contact with children, foreign traveling, sexual promiscuity, addictions, and washing hands before eating. Housing characteristics included type of flooring, form of elimination of excretes, and crowding.

\section{Detection of anti-CMV IgG and IgM antibodies}

A blood sample from each participant was collected. After centrifugation of blood samples, serum samples were obtained and kept frozen until analyzed. Sera were tested for anti-CMV IgG antibodies by the commercially available enzyme linked fluorescent assay (ELFA) "VIDAS CMV IgG" (BioMerieux, Marcy l'Etoile, France). This test allows qualitative and quantitative detection of anti-CMV IgG antibodies. Anti-CMV IgG antibody levels were expressed as Arbitrary Units (AU)/mL. A cut-off of $6 \mathrm{AU} / \mathrm{mL}$ was used for seropositivity. In addition, all serum samples were examined by the commercially available ELFA "VIDAS CMV IgM" (BioMerieux, France). This assay is a qualitative test for detection of anti-CMV IgM antibodies. 


\section{Statistical analysis}

We performed the statistical analysis with the aid of the software Epi Info 7 and SPSS version 15.0. For calculation of the sample size, we used a value of 20,000 as a population size from which the sample was selected, a reference seroprevalence of $89.2 \%$ [12] as expected frequency of the factor under study, $5 \%$ of confidence limits, a design effect of 1.0 , one cluster, and a confidence level of $95 \%$. The result of the calculation was 147 subjects. The Pearson's Chi-square test or the Fisher exact test (when cell values were $<5$ ) was used to compare the frequencies among groups. The association between the characteristics of the women and CMV seropositivity was assessed by bivariate and multivariate analyses. To avoid bias in the process of data analysis, clinical characteristics were analyzed separated from socio-demographic, behavioral and housing characteristics. Variables with a $\mathrm{P}$ value $\leq 0.20$ obtained in the bivariate analysis were further examined by logistic regression using the Enter method. Odds ratios (ORs) and 95\% confidence intervals (CIs) were calculated, and a P value $<0.05$ was considered statistically significant.

\section{Ethical aspects}

The Ethical Committee of Instituto de Servicios de Salud del Estado de Aguascalientes approved this project. The purpose and procedures of this study were explained to pregnant women. Participation in the study was voluntary, and a written informed consent was obtained from all participants and from the next of kin of minor participants.

\section{Results}

Anti-CMV IgG antibodies were detected in 259 (89.6\%) of the 289 pregnant women studied. Of the 259 anti-CMV IgG positive women, $16(5.5 \%)$ had $\operatorname{IgG}$ levels higher than 150 $\mathrm{AU} / \mathrm{mL}, 40$ (13.8\%) between 100 and $150 \mathrm{AU} / \mathrm{mL}$, and 203 $(70.2 \%)$ between 6 and $99 \mathrm{AU} / \mathrm{mL}$. Individual values of anti-CMV IgG antibodies obtained in the 289 pregnant women studied are shown in Figure 1. None of the 289 pregnant women were positive for anti-CMV IgM antibodies. General socio-demographic characteristics of the pregnant women surveyed are shown in Table 1. None of the socio-demographic characteristics of pregnant women including age, ethnic group, birthplace, residence, occupation, educational level, and socioeconomic status was associated $(\mathrm{P}>0.05)$ with seropositivity to CMV by bivariate analysis.

With respect to clinical characteristics, seroprevalence of CMV infection did not vary in pregnant women regardless of their health status, presence or history of lymphadenopathy, frequent headaches and impairments of memory, vision or hearing. Table 2 shows a correlation of clinical data of pregnant women and seroprevalence of CMV infection. Seroprevalence of $\mathrm{CMV}$ infection was significantly lower $(\mathrm{P}=$ $0.03)$ in pregnant women with reflex impairment (5/8: $62.5 \%)$ than in those without this clinical feature (246/272: 90.4\%). In addition, seroprevalence of CMV infection did not vary with history of blood transfusions, hepatitis, or surgery (Table 2). None of the pregnant women had a history of organ transplantation. Concerning obstetric history, seroprevalence of CMV infection was similar in pregnant women regardless of their month of pregnancy, number of deliveries, cesarean sections or miscarriages. In contrast, seroprevalence of CMV infection was significantly higher $(\mathrm{P}=0.03)$ in pregnant women with 2 - 9 pregnancies (140/150: $93.3 \%)$ than in those with only one pregnancy (119/139: 86.2\%).

Concerning behavioral characteristics, seroprevalence of $\mathrm{CMV}$ infection did not vary in pregnant women regardless of their history of foreign traveling, sexual promiscuity, addictions or washing hands before eating. Table 3 shows a correlation of behavioral characteristics of pregnant women and CMV infection. In contrast, seroprevalence of CMV infection was significantly higher $(\mathrm{P}=0.03)$ in pregnant women with contact with children (241/266: $90.6 \%)$ than in those without this behavioral characteristic (14/19: 73.7\%). None of the housing characteristics including type of flooring, form of elimination of excretes, and crowding was associated with CMV infection. Logistic regression analysis of socio-demographic and behavioral variables with $P$ values $\leq 0.20$ obtained by bivariate analysis (occupation, contact with children, and type of flooring at home) showed that CMV infection was associated only with contact with children $(\mathrm{OR}=3.56$; 95\% CI: 1.17 - 10.84; $\mathrm{P}=0.02$ ).

Further analysis to determine the association between high $(>150 \mathrm{AU} / \mathrm{mL})$ anti-CMV IgG antibody levels and sociodemographic, clinical, behavioral, and housing characteristics from the pregnant women was performed. Bivariate analysis of these characteristics showed that the presence of high CMV $\mathrm{IgG}$ antibody levels in pregnant women was associated $(\mathrm{P}<$ 0.05 ) only with the variables including no washing hands before eating, health status, occupation and low education. Logistic regression analysis of socio-demographic and behavioral variables associated with high CMV IgG antibody levels obtained by bivariate analysis showed that high antibody levels were associated only with no washing hands before eating (OR $=0.17 ; 95 \%$ CI: $0.05-0.63 ; \mathrm{P}=0.007)$.

\section{Discussion}

Very little is known about the seroepidemiology of CMV infection in pregnant women in Mexico. We are aware of only two studies on the seroprevalence of CMV infection in pregnant women in Mexico. In a previous study, we reported the seroepidemiology of CMV infection in pregnant women in the northern Mexican city of Durango [11]. In another Mexican study, researchers reported the seroprevalence of CMV infection in pregnant women in Morelia, Michoacan [13]. Therefore, in the present study, we investigated the seroprevalence and correlates of CMV infection in pregnant women in the central Mexican city of Aguascalientes. We found an $89.6 \%$ seroprevalence in pregnant women in Aguascalientes City. This seroprevalence is higher than the $65.6 \%$ seroprevalence found in pregnant women in Durango City [11]. The seroprevalence 
Table 1. Socio-Demographic Characteristics of Pregnant Women and Prevalence of CMV Infection

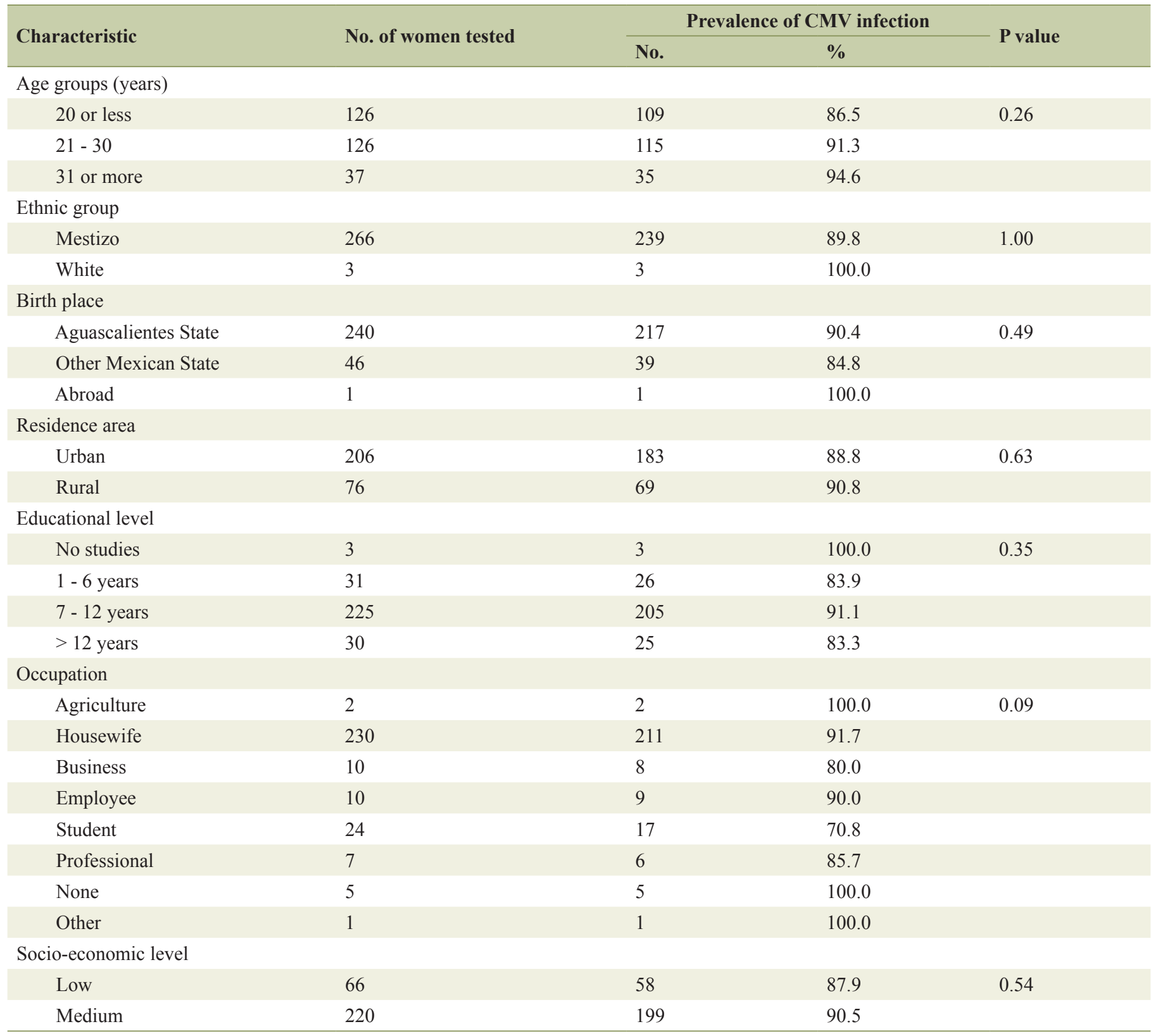

of CMV infection in women in Aguascalientes is comparable with a $92.6 \%$ seroprevalence of CMV infection reported in healthy women at reproductive age in Cuernavaca City in central Mexico [14], and an 89.2\% seroprevalence found in a national survey in subjects aged 1 - 70 years old in Mexico [12]. In an international context, the seroprevalence found in pregnant women in Aguascalientes is as high as the 88.5$100 \%$ seroprevalences reported in pregnant women in Ethiopia [15], China [16], Cuba [17], Iran [18], Brazil [19], Palestine [20], Turkey [21], and Nigeria [22]. On the other hand, the seroprevalence found in pregnant women in Aguascalientes is higher than the 42.3-66\% seroprevalences reported in pregnant women in Germany [23], France [24], Poland [25], Japan [26], and Norway [27]. Therefore, comparison of the seroprevalence found in our study with those reported in other countries suggests that seroprevalence of CMV infection in pregnant women in Aguascalientes City could be placed in a high position of endemicity. However, interpretation of this comparison of seroprevalences should be cautious since different laboratory methods for detection of anti-CMV IgG antibodies were used among the studies. In the present study, we used ELFA, whereas in the other studies, laboratory tests for detection of anti-CMV IgG antibodies other than ELFA were used.

We searched for seroprevalence association with sociodemographic, clinical, behavioral, and housing characteristics from the pregnant women. Logistic regression analysis showed 
Table 2. Bivariate Analysis of Clinical Data and Infection With CMV in Pregnant Women

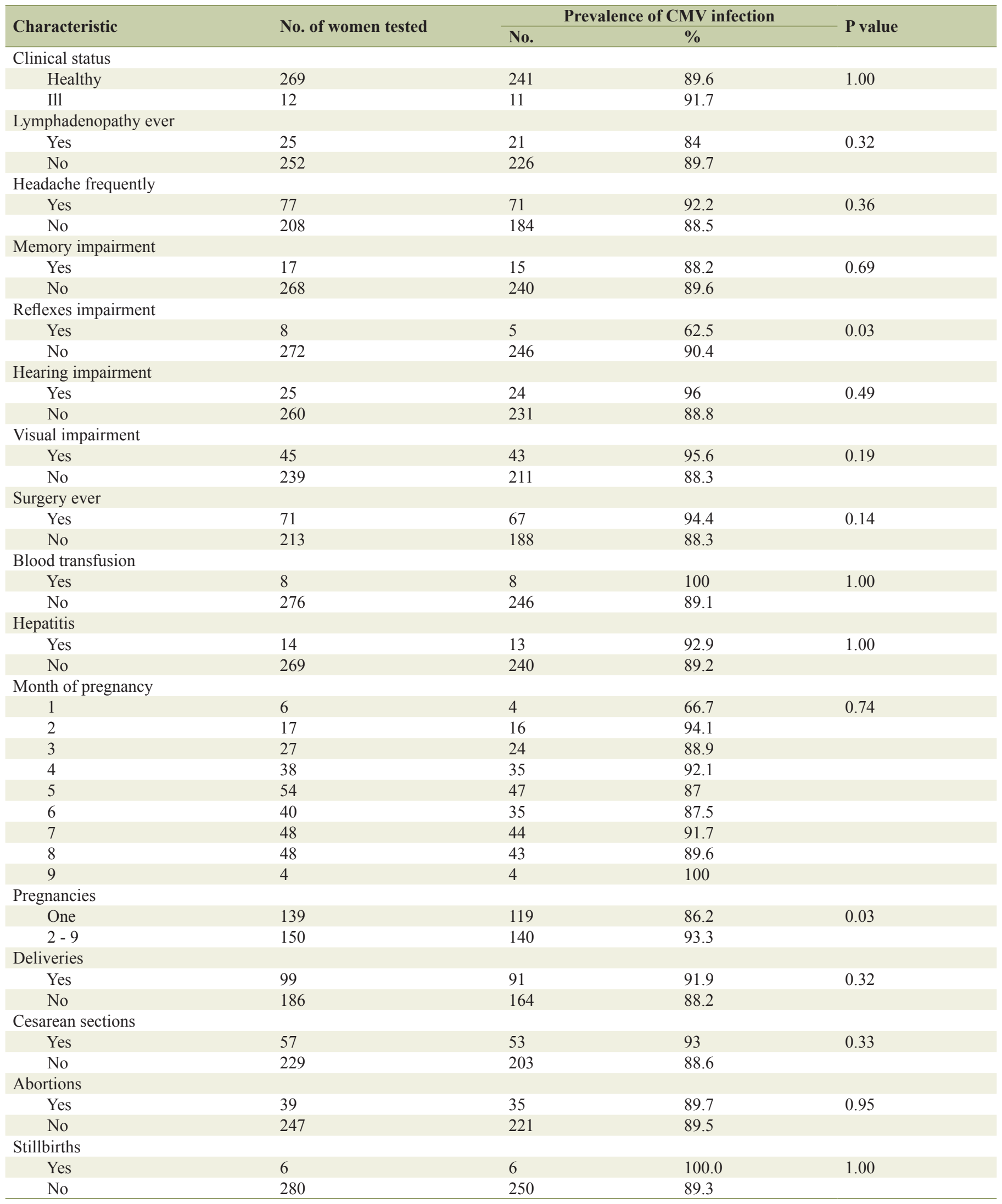


Table 3. Bivariate Analysis of Behavioral Characteristics of Pregnant Women and CMV Infection

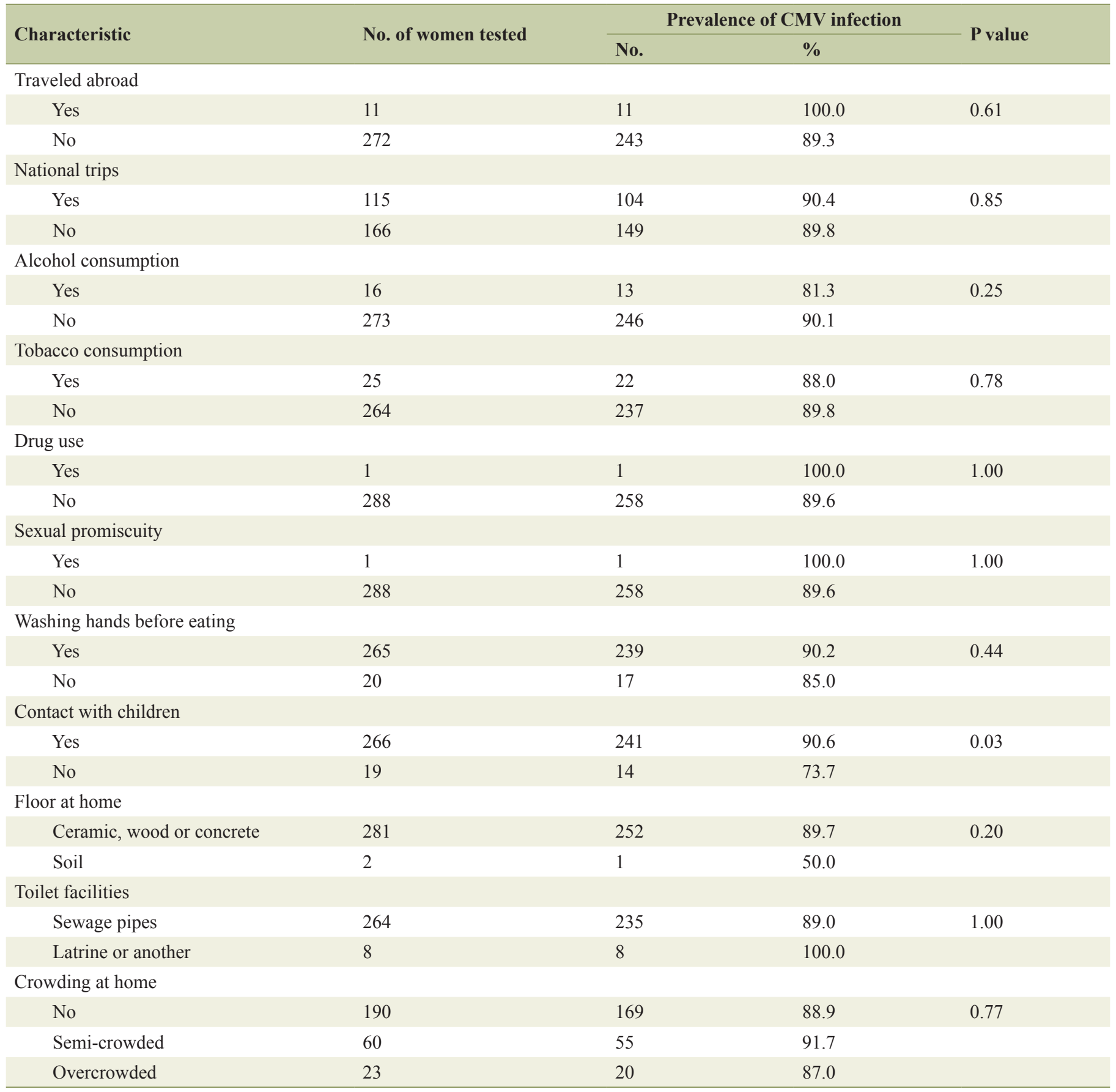

that seropositivity to CMV was associated with contact with children. Young children are considered the key transmission risk of CMV infection for pregnant women [4, 15, 28]. However, the association between seropositivity to CMV and contact with children found in the present study conflicts with that reported in a study of pregnant women in Poland where researchers found that occupational risk related to contact with children was not related with the prevalence of anti-CMV IgG antibodies [25]. Logistic regression analysis also showed in our study that high anti-CMV IgG levels were negatively associated with washing hands before eating. To the best of our knowledge, this is the first report of a negative association between high anti-CMV IgG antibodies and washing hand before eating. In a study of CMV survival and transferability and the effectiveness of common hand-washing agents against CMV on live human hands, viable CMV was recovered from 4/20 hands $10 \mathrm{~min}$ after diaper wipe cleansing [29]. Hand washing is considered an effective means of preventing the acquisition 
of CMV by pregnant women and by individuals who care for children [30]. Our result further stresses the protective role of washing hands to avoid CMV infection in pregnant women. Furthermore, in our study, seroprevalence of CMV infection was significantly higher in pregnant women with 2 - 9 pregnancies than in those with only one pregnancy. This is likely due to contact with children since women with several pregnancies have contact with their own children whereas women at their first pregnancy do not have still contact with their children. In the current study, seroprevalence of CMV infection was significantly lower in pregnant women with reflex impairment than in those without this clinical feature. This result suggests that CMV infection did not play an important role in reflex impairment in the pregnant women studied. Based on our results of CMV IgG and IgM antibodies tests, all seropositive women had latent infections. Known factors for CMV infection including age [26], socio-economic status [14, 31], sexual promiscuity, education [14], and blood transfusion [32] were not found associated with CMV infection in our study.

The present study has some limitations. The sample size of pregnant women was relatively small. Only pregnant women attending in one of several health institutions in Aguascalientes City were studied. No pregnant women attending in private clinics were studied. Therefore, further studies to determine the epidemiology of CMV infection in pregnant women in Aguascalientes should be conducted.

\section{Conclusions}

We found a high endemicity of CMV infection in pregnant women in Aguascalientes City, Mexico. Factors associated with CMV infection found in this study may be useful for an optimal planning of preventive measures against CMV exposure in pregnant women.

\section{Acknowledgments}

We acknowledge the kind support of the personnel of Instituto de Servicios de Salud, Aguascalientes, Mexico.

\section{Competing Interests}

The authors declare that they have no competing interests.

\section{Financial Support}

This study was financially supported by Juarez University of Durango State.

\section{References}

1. Zamora MR. DNA viruses (CMV, EBV, and the herpesviruses). Semin Respir Crit Care Med. 2011;32(4):454-470.
2. Drebber U, Hardt A, Dienes HP, Odenthal M. [Cytomegalovirus. Pathological-anatomical manifestations and detection methods]. Pathologe. 2011;32(5):418-427.

3. Davis NL, King CC, Kourtis AP. Cytomegalovirus infection in pregnancy. Birth Defects Res. 2017;109(5):336346.

4. Johnson J, Anderson B, Pass RF. Prevention of maternal and congenital cytomegalovirus infection. Clin Obstet Gynecol. 2012;55(2):521-530.

5. Cannon MJ, Hyde TB, Schmid DS. Review of cytomegalovirus shedding in bodily fluids and relevance to congenital cytomegalovirus infection. Rev Med Virol. 2011;21(4):240-255.

6. Saldan A, Forner G, Mengoli C, Gussetti N, Palu G, Abate D. Testing for Cytomegalovirus in Pregnancy. J Clin Microbiol. 2017;55(3):693-702.

7. Leruez-Ville M, Ville Y. Fetal cytomegalovirus infection. Best Pract Res Clin Obstet Gynaecol. 2017;38:97-107.

8. Wieringa JW, de Vries JJ, Murk JL. [Congenital CMV infections]. Ned Tijdschr Geneeskd. 2013;157(41):A6250.

9. Allain JP, Stramer SL, Carneiro-Proietti AB, Martins ML, Lopes da Silva SN, Ribeiro M, Proietti FA, et al. Transfusion-transmitted infectious diseases. Biologicals. 2009;37(2):71-77.

10. Azevedo LS, Pierrotti LC, Abdala E, Costa SF, Strabelli TM, Campos SV, Ramos JF, et al. Cytomegalovirus infection in transplant recipients. Clinics (Sao Paulo). 2015;70(7):515-523.

11. Alvarado-Esquivel C, Hernandez-Tinoco J, Sanchez-Anguiano LF, Ramos-Nevarez A, Cerrillo-Soto SM, Estrada-Martinez S, Martinez-Ramirez L, et al. Seroepidemiology of cytomegalovirus infection in pregnant women in Durango City, Mexico. BMC Infect Dis. 2014;14:484.

12. Conde-Glez C, Lazcano-Ponce E, Rojas R, DeAntonio R, Romano-Mazzotti L, Cervantes Y, Ortega-Barria E. Seroprevalences of varicella-zoster virus, herpes simplex virus and cytomegalovirus in a cross-sectional study in Mexico. Vaccine. 2013;31(44):5067-5074.

13. Gonzalez-Garcia CL, Reyes-Mendez MA, Ortega-Pierres LE, Rodriguez-Sanchez AP, Sandoval-Guido V, Sereno-Colo JA. [Seroprevalence and detection of primary infection by cytomegalovirus with IgG avidity test during the first quarter of pregnancy]. Salud Publica Mex. 2014;56(6):619-624.

14. Echaniz-Aviles G, Tamayo-Legorreta E, Cruz-Valdez A, Rangel-Flores H, Hernandez-Nevarez P, Gatica-Marquina R, Calderon-Jaimes E. [Prevalence of antibodies against cytomegalovirus in women of reproductive age]. Salud Publica Mex. 1993;35(1):20-26.

15. Yeshwondm M, Balkachew N, Delayehu B, Mekonen G. Seroepidemiology Study of cytomegalovirus and rubella among pregnant women at St. Paul's Hospital Millennium Medical College, Addis Ababa, Ethiopia. Ethiop J Health Sci. 2016;26(5):427-438.

16. Jin $\mathrm{Q}, \mathrm{Su}$ J, Wu S. Cytomegalovirus infection among pregnant women in Beijing: seroepidemiological survey and intrauterine transmissions. J Microbiol Biotechnol. 2017;27(5):1005-1009.

17. Correa CB, Kouri V, Verdasquera D, Martinez PA, Alva- 
rez A, Aleman Y, Perez L, et al. HCMV seroprevalence and associated risk factors in pregnant women, Havana City, 2007 to 2008. Prenat Diagn. 2010;30(9):888-892.

18. Erfanianahmadpoor M, Nasiri R, Vakili R, Hassannia T. Seroprevalence, transmission, and associated factors of specific antibodies against cytomegalovirus among pregnant women and their infants in a regional study. Saudi Med J. 2014;35(4):360-364.

19. Yamamoto AY, Castellucci RA, Aragon DC, Mussi-Pinhata MM. Early high CMV seroprevalence in pregnant women from a population with a high rate of congenital infection. Epidemiol Infect. 2013;141(10):2187-2191.

20. Neirukh T, Qaisi A, Saleh N, Rmaileh AA, Zahriyeh EA, Qurei L, Dajani F, et al. Seroprevalence of Cytomegalovirus among pregnant women and hospitalized children in Palestine. BMC Infect Dis. 2013;13:528.

21. Aynioglu A, Aynioglu O, Altunok ES. Seroprevalence of Toxoplasma gondii, rubella and Cytomegalovirus among pregnant females in north-western Turkey. Acta Clin Belg. 2015;70(5):321-324.

22. Akinbami AA, Rabiu KA, Adewunmi AA, Wright KO, Dosunmu AO, Adeyemo TA, Adediran A, et al. Seroprevalence of cytomegalovirus antibodies amongst normal pregnant women in Nigeria. Int J Womens Health. 2011;3:423-428.

23. Enders G, Daiminger A, Lindemann L, Knotek F, Bader U, Exler S, Enders M. Cytomegalovirus (CMV) seroprevalence in pregnant women, bone marrow donors and adolescents in Germany, 1996-2010. Med Microbiol Immunol. 2012;201(3):303-309.

24. N'Diaye DS, Yazdanpanah Y, Krivine A, Andrieu T, Rozenberg F, Picone O, Tsatsaris V, et al. Predictive factors of cytomegalovirus seropositivity among pregnant women in Paris, France. PLoS One. 2014;9(2):e89857.
25. Wujcicka W, Gaj Z, Wilczynski J, Sobala W, Spiewak E, Nowakowska D. Impact of socioeconomic risk factors on the seroprevalence of cytomegalovirus infections in a cohort of pregnant Polish women between 2010 and 2011. Eur J Clin Microbiol Infect Dis. 2014;33(11):1951-1958.

26. Shigemi D, Yamaguchi S, Otsuka T, Kamoi S, Takeshita T. Seroprevalence of cytomegalovirus IgG antibodies among pregnant women in Japan from 2009-2014. Am J Infect Control. 2015;43(11):1218-1221.

27. Barlinn R, Vainio K, Samdal HH, Nordbo SA, Nokleby $\mathrm{H}$, Dudman SG. Susceptibility to cytomegalovirus, parvovirus B19 and age-dependent differences in levels of rubella antibodies among pregnant women. J Med Virol. 2014;86(5):820-826.

28. Pass RF, Little EA, Stagno S, Britt WJ, Alford CA. Young children as a probable source of maternal and congenital cytomegalovirus infection. N Engl J Med. 1987;316(22):1366-1370.

29. Stowell JD, Forlin-Passoni D, Radford K, Bate SL, Dollard SC, Bialek SR, Cannon MJ, et al. Cytomegalovirus survival and transferability and the effectiveness of common hand-washing agents against cytomegalovirus on live human hands. Appl Environ Microbiol. 2014;80(2):455461 .

30. Onorato IM, Morens DM, Martone WJ, Stansfield SK. Epidemiology of cytomegaloviral infections: recommendations for prevention and control. Rev Infect Dis. 1985;7(4):479-497.

31. Knowles SJ, Grundy K, Cahill I, Cafferkey MT, Geary M. Low cytomegalovirus sero-prevalence in Irish pregnant women. Ir Med J. 2005;98(7):210-212.

32. Maingi Z, Nyamache AK. Seroprevalence of Cytomegalo Virus (CMV) among pregnant women in Thika, Kenya. BMC Res Notes. 2014;7:794. 